\title{
Rediscovering IgA
}

\author{
NJ Mantis ${ }^{1}$
}

$\underset{\text { in }}{\mathrm{i}}$

xtraordinary amounts of immunoglobulin A (IgA) are produced in the intestinal mucosa and secreted into the human gastrointestinal tract each day. IgA production is driven largely in response to mucosal antigens encountered by gut-associated lymphoid tissue. Although the actual specificity and breadth of the antigenic repertoire of secretory $\operatorname{IgA}(\mathrm{SIgA})$ are unknown, it is clear that secretory antibodies are directed against at least two broad classes of antigens. The first is associated with enteric pathogens and their virulence determinants, such as toxins. Evidence for this is provided by fecal IgA-reactivity profiles from individuals who reside in regions where enteric diseases are endemic. ${ }^{1}$

The second broad class of antigens recognized by SIgA is associated with the intestinal microbiota (or commensal microflora). In experimental animal models (e.g., gnotobiotic mice), commensal bacteria are potent inducers of secretory antibodies; in humans, it is estimated that between 25 and $75 \%$ of intestinal bacteria are coated with SIgA. 2,3 Additionally, there is evidence from mice that secretory antibodies play an important role in shaping the composition of the intestinal microbiota, which in turn can influence epithelial antimicrobial defenses and enhance resistance to the intestinal pathogens. $^{4,5}$

Thus, by virtue of its ability to neutralize enteric pathogens and shape the commensal microbiota, SIgA plays a profound role in intestinal homeostasis.
This conclusion may come as no surprise to the readers of Mucosal Immunology, but not everyone will be familiar with the extraordinary advances over the past several years in our understanding of IgA's multifaceted roles in mucosal and systemic immunity. In fact, IgA is now experiencing a renaissance of sorts, as it is becoming increasingly evident that human health is inextricably linked to the gut microbiota, intestinal homeostasis, and mucosal immunity. $\operatorname{IgA}$ is at the center of this dynamic.

This special-focus issue of the journal is designed to provide the reader with an up-to-date and in-depth review of key facets of IgA biology. Jenny Woof and Michael Russell provide a detailed description of the structure-function relationships of human IgA subclasses and implications for mucosal immunity and IgA-based therapeutics. ${ }^{6}$ FinnEirik Johansen and Charlotte Kaetzel summarize recent advances in elucidating environmental factors that influence transepithelial IgA transport by the polymeric immunoglobulin receptor (pIgR). ${ }^{7}$ In particular, there appears to be a positive-feedback loop in which pIgR expression (and therefore SIgA levels) is regulated by the epithelium in response to Toll-like receptor stimulation by commensal microflora. Local inflammatory cytokine levels elicited in response to infections further influence pIgR expression.

Nicolas Rol, Blaise Cothésy, and I describe the extraordinarily diverse functions that have been attributed to SIgA

${ }^{1}$ Guest Editor

doi:10.1038/mi.2011.42 
in the intestinal lumen. ${ }^{8}$ Certain SIgA antibodies have been shown to directly quench bacterial virulence, whereas others mediate the retrotranslocation and uptake of SIgA-immune complexes by mucosal dendritic cells and result in the subsequent downregulation of proinflammatory responses normally associated with pathogens and allergic antigens.

Finally, Jantine Bakema and Marjolein van Egmond detail the interactions of IgA with the human Fc $\alpha$ RI receptor (CD89) expressed on neutrophils, eosinophils, monocytes, and several macrophageand monocyte-derived dendritic cell subsets. ${ }^{9}$ The functional outcome of Fc $\alpha$ RI engagement is dictated by the nature of IgA-whereas monomeric IgA triggers inhibitory signals, aggregated IgA results in crosslinking of CD89 and induction of phagocytosis and proinflammatory cytokine release. This special issue does not cover IgA class switching or homing of IgA-secreting cells, as these topics have recently been reviewed elsewhere. ${ }^{10,11}$

It is my hope that this issue of Mucosal Immunology will serve as an important reference for the research community as the "rediscovery" of IgA continues.
(C) 2011 Society for Mucosal Immunology

\section{REFERENCES}

1. Pasetti, M.F., Simon, J.K., Sztein, M.B. \& Levine, M.M. Immunology of gut mucosal vaccines. Immunol. Rev. 239, 125-148 (2011).

2. Talham, G.L., Jiang, H.Q., Bos, N.A. \& Cebra, J.J. Segmented filamentous bacteria are potent stimuli of a physiologically normal state of the murine gut mucosal immune system. Infect. Immun. 67, 1992-2000 (1999).

3. van der Waaij, L.A., Limburg, P.C., Mesander, G. \& van der Waaij, D. In vivo IgA coating of anaerobic bacteria in human faeces. Gut 38, 348-354 (1996).

4. Ivanov, I.I. et al. Induction of intestinal Th17 cells by segmented filamentous bacteria. Cell 139, 485-498 (2009).

5. Suzuki, K. et al. Aberrant expansion of segmented filamentous bacteria in IgA-deficient gut. Proc. Natl. Acad. Sci. USA 101, 1981-1986 (2004).

6. Woof, J.M. \& Russell, M.W. Structure and function relationships in IgA. Mucosal Immunol. 4, 590-597 (2011).

7. Johansen, F.-E. \& Kaetzel, C.S. Regulation of the polymeric immunoglobulin receptor and IgA transport: new advances in environmental factors that stimulate plgR expression and its role in mucosal immunity. Mucosal Immunol. 4, 598-602 (2011).

8. Mantis, N.J., Rol, N. \& Corthésy, B. Secretory IgA's complex roles in immunity and mucosal homeostasis in the gut. Mucosal Immunol. 4, 603-611 (2011).

9. Bakema, J.E. \& van Egmond, M. The human immunoglobulin A Fc receptor FcoRl: a multifaceted regulator of mucosal immunity. Mucosal Immunol. 4, 612-624 (2011).

10. Cerutti, A. The regulation of IgA class switching. Nat. Rev. Immunol. 8, 421-434 (2008).

11. Mora, J.R. \& von Andrian, U.H. Differentiation and homing of IgA-secreting cells. Mucosal Immunol. 1 96-109 (2008). 